NUTRITION Les bases de la politique nutritionnelle de santé publique en France : le constat et les recommandations du HCSP

\title{
NUTRITION Les bases de la politique nutritionnelle de santé publique en France : le constat et les recommandations du HCSP
}

Oléagineux, Corps Gras, Lipides. Volume 8, Numéro 1, 7-12, Janvier - Février 2001, La filière

\begin{abstract}
Auteur(s) : Serge HERCBERG, Unité de surveillance et d'épidémiologie nutritionnelle (USEN), Institut scientifique et technique de la nutrition et de I'alimentation, Conservatoire national des arts et métiers, 5, rue Vertbois, 75003 Paris, France.
\end{abstract}

Résumé : La mise en place d'une politique nutritionnelle apparaît comme une grande priorité de santé publique en France, comme dans l'ensemble de l'Europe. Outre les immenses enjeux humains, sociaux et économiques, les relations entre l'alimentation et la santé ont comme particularité : de concerner l'ensemble de la population; de reposer sur des connaissances scientifiques bien documentées; de concerner des facteurs de risque (ou de protection) bien identifiés sur lesquels il est possible d'intervenir ; d'avoir un impact important sur l'incidence des grandes maladies chroniques; d'impliquer, autour du consommateur, de multiples acteurs du champ de la nutrition ; et d'avoir des références en termes de faisabilité. Pour toutes ces raisons, il apparaît aujourd'hui indispensable de faire, en France, de la promotion de la nutrition et de l'activité physique, un grand " programme national nutrition-santé ", impliquant l'ensemble des acteurs concernés et disposant des moyens à la hauteur de ses ambitions. C'est la conclusion du groupe de travail ayant rédigé le rapport "Pour une politique nutritionnelle de santé publique en France : enjeux et propositions ", sous l'égide du HCSP.

Mots-clés : politique nutritionnelle, alimentation, santé publique, cancer, maladies cardiovasculaires, obésité, ostéoporose.

Summary : The elaboration of a nutritional policy is one of the most important public health priorities in France and throughout Europe. In addition to immense human, social and economic stakes, the relationship between nutrition and health is unique in that: it concerns the entire population; it is based on thoroughly documented scientific knowledge; it involves well known risk (and protective) factors upon which it is possible to intervene; it has a considerable impact upon the incidence of the most frequently encountered chronic diseases; it involves the participation not only of the consumer but of all persons working in the nutritional field; and its feasibility has been demonstrated. For all these reasons, it has now become indispensable in France to promote nutrition and physical activity by setting up 
a wide-scale "National Nutrition and Health Program" involving all of the concerned parties and having at its disposal sufficient means to carry it out.

This is the conclusion of the Work-Study Group, which has drawn up a report entitled "For a public health nutritional policy in France: stakes and propositions", under the auspices of the HCSP.

Keywords : nutritional policy, foods, public health, cancer, cardiovascular diseases, obesity, osteoporosis.

\section{ARTICLE}

Le choix de la nutrition, comme thème marquant dans le domaine de la santé de la présidence française de l'Union européenne, a suscité au cours des deux dernières années une réflexion en profondeur, au sein de la Direction générale de la Santé (DGS), sur la nécessité de développer, en France, une politique nutritionnelle de santé publique. Près de quarante personnalités, scientifiques, chercheurs, représentants des instances administratives (Santé, Éducation nationale et Agriculture, InVS, Afssa), médecins hospitaliers, industriels de l'agroalimentaire ont contribué à un rapport élaboré par un groupe de travail mandaté par le Haut comité de la santé publique (HCSP) destiné à la secrétaire d'État à la Santé et récemment publié aux éditions ENSP [1]. Ce rapport de 250 pages fait un point sur le contexte des relations entre l'alimentation et la santé et, surtout, propose une série de recommandations pour la mise en place d'une politique nutritionnelle de santé publique en France.

Ce texte reprend les grandes lignes du rapport présenté au secrétaire d'État à la Santé en juin 2000 et publié sous forme d'un ouvrage en septembre 2000 [1].

\section{Constat}

\section{Enjeux de santé publique}

L'épidémiologie des problèmes de santé dans lesquels des facteurs nutritionnels sont impliqués permet de mesurer l'importance des enjeux humains, sociaux et économiques.

* Les maladies cardiovasculaires constituent actuellement la première cause de mortalité en France, à l'origine de près de 170000 décès chaque année ( $32 \%$ des décès, dont près de 10 $\%$ surviennent avant 65 ans).

* Les tumeurs malignes représentent $29 \%$ de l'ensemble des décès chez l'homme et $23 \%$ des décès chez la femme. Le nombre de nouveaux cas est estimé à 240000 par an.

* L'obésité concerne 7 à $10 \%$ des adultes et 10 à $12,5 \%$ des enfants de 5 à 12 ans. Sa prévalence augmente, chez les enfants, de façon dramatique depuis quelques années.

* L'ostéoporose, à l'origine d'une fragilité osseuse exposant les patients à un risque de fractures, concernerait $10 \%$ des femmes à 50 ans, $20 \%$ à 60 ans et $40 \%$ à 75 ans. 
* La prévalence du diabète, tous âges confondus, est estimée entre 2 et 2,5\%.

* Près d'un adulte sur 5 présente une cholestérolémie supérieure à 2,50 g/l.

En dehors de leurs conséquences sur le plan humain, ces pathologies ont un coût économique considérable : les coûts directs et indirects des cardiopathies ischémiques sont estimés à 30 milliards de francs/an ; ceux attri- buables à l'obésité atteindraient près de 12 milliards de francs/an ; les seules complications fracturaires du col fémoral en rapport avec l'ostéoporose coûtent chaque année entre 4 et 7 milliards de francs, etc.

\section{Consommation alimentaire et état nutritionnel en France}

Les habitudes alimentaires ont beaucoup plus évolué au cours des cinquante dernières années qu'au cours des siècles précédents, sous l'influence de multiples déterminants qui sont passés en revue. La consommation de produits céréaliers, de légumes secs et de féculents a considérablement chuté depuis le début des années 50 , mais semble désormais se stabiliser. À l'inverse, on constate une augmentation de la consommation de produits sucrés, de fruits et légumes, cette dernière étant cependant en diminution depuis quelques années. Augmentation également de la consommation de viandes, de poissons, d'œufs et de produits laitiers avec toutefois, pour les viandes et le lait sous forme liquide, un recul récent de leur consommation. La consommation des matières grasses visibles a considérablement augmenté au cours des dernières décennies mais a tendance à se stabiliser depuis les dix dernières années. La consommation de sel semble excessive dans une fraction importante de la population.

Ces évolutions de l'alimentation au cours des dernières décennies se traduisent sur le plan nutritionnel par:

* une réduction globale de l'apport énergétique, en réponse à la diminution des dépenses énergétiques ;

* une diversification importante des apports alimentaires ;

* une modification de la contribution des différents macronutriments aux apports énergétiques :

- diminution de la part des glucides complexes et augmentation de la part des sucres simples (saccharose),

- augmentation de la part des protéines d'origine animale et diminution de la part des protéines d'origine végétale,

- augmentation de la part des lipides, notamment invisibles et saturés ;

* une réduction des apports en de nombreux micronutriments en rapport avec, d'une part, la réduction globale des apports énergétiques et, d'autre part, la modification structurelle de la ration caractérisée aujourd'hui par une part de plus en plus importante d'aliments fournissant des calories "vides » (aliments contenant des sucres simples, mais sans apport 
de micronutriments). Ce phénomène a contribué à réduire la " densité " en micronutriments de l'alimentation, c'est-à-dire la concentration en vitamines et minéraux par unité d'énergie.

Ces profondes modifications comportent, sur le plan nutritionnel et sur le plan de la santé, des aspects positifs et des aspects négatifs, des avantages et des inconvénients. L'alimentation est incontestablement plus variée, plus diversifiée qu'autrefois, ce qui est favorable à la santé. Mais, si les grands problèmes de carence sévère ont disparu, l'évolution de l'alimentation en France a eu quelques conséquences négatives en termes de facteurs de risque de certaines maladies chroniques. Sont passés en revue dans le rapport les résultats des principales études nutritionnelles visant à préciser les apports alimentaires et le statut nutritionnel des français.

\section{Facteurs nutritionnels impliqués dans le déterminisme des maladies chroniques}

Au cours des trente dernières années, de très nombreux travaux scientifiques sur modèles cellulaires, organes, animaux, chez l'homme sain et malade ainsi qu'au niveau de populations ont mis en évidence, avec des degrés de certitude différents, le rôle de facteurs nutritionnels (excès ou insuffisance) dans le déterminisme de nombreuses maladies. Si, dans de nombreux cas, les arguments disponibles ne permettent pas (encore) d'affirmer la réalité du lien entre le facteur nutritionnel suspecté et certaines maladies, dans d'autres cas, au contraire, la convergence des arguments est telle qu'elle fait l'objet d'un consensus international. Dans le rapport sont passés en revue les arguments mécanistiques, cliniques et épidémiologiques permettant de dresser l'état des connaissances sur le rôle des facteurs alimentaires et nutritionnels impliqués dan le déterminisme des cancers, des maladies cardio-vasculaires, de l'obésité, de l'ostéoporose et les problèmes nutritionnels spécifiques à des populations particulières (enfants et adolescents, femmes enceintes et allaitantes, personnes âgées, personnes hospitalisées, sujets en situation de précarité, population étrangère et immigrée, personnes souffrant de troubles de la conduite alimentaire ou suivant des régimes restrictifs, personnes souffrant d'allergie alimentaire).

\section{Acteurs, structures et organisations impliqués dans le champ de la nutrition}

Le rapport présente les nombreux acteurs, structures et organisations impliqués dans le champ de la nutrition. Un dispositif institutionnel élaboré organise, surveille, expertise, réglemente et sécurise. II repose sur les nombreux ministères concernés par l'alimentation et la nutrition, principalement ceux de la Santé, de l'Agriculture et des Finances. Le paysage institutionnel français a connu d'importantes transformations au cours des années récentes avec la création, en 1998, de l'Agence française de sécurité sanitaire des aliments (Afssa), qui a repris les fonctions de nombreuses commissions et organismes existants, et la mise en place en 2000 de I'Usen, Unité de surveillance et d'épidémiologie nutritionnelle au sein de I'Institut national de veille sanitaire (InVS), et la refonte du Conseil national de l'alimentation (CNA).

Différents professionnels et structures (médecins, paramédicaux, professionnels de l'industrie agro-alimentaire, secteur de l'éducation nutritionnelle, diététiciens, structures associatives spécialisées) interviennent dans le domaine des interrelations entre nutrition et 
santé.

La recherche fournit les éléments de base qui permettent d'identifier les facteurs alimentaires et nutritionnels constituant des facteurs de risque ou de protection vis-à-vis des maladies chroniques.

De multiples acteurs économiques assurent la production, la transformation et la distribution des aliments.

Enfin, le consommateur occupe bien entendu une position centrale dans ce paysage, puisque c'est pour lui que fonctionne l'ensemble de ce dispositif, et que, même s'il est plus ou moins fortement influencé par son environnement, c'est lui qui, en dernier lieu, fait le choix de sa consommation alimentaire.

\section{Recommandations}

Dans un domaine où des possibilités d'intervention préventives existent, le rapport insiste sur le fait que la volonté politique doit être à la hauteur des enjeux de santé publique aujourd'hui bien identifiés. Seul un véritable " programme national nutrition-santé ", ambitieux et rigoureux sur le plan méthodologique, fondé sur des objectifs précis, associant le plus grand nombre d'acteurs et faisant l'objet d'un pilotage par une équipe compétente peut permettre une évolution significative de la situation actuelle.

\section{Objectifs nutritionnels prioritaires de santé publique et objectifs spécifiques}

\section{Les objectifs nutritionnels prioritaires de santé publique (encadré)}

Les objectifs ont été définis en 1999 par un comité d'experts coordonné par Serge Hercberg et Arnaud Basdevant dans un rapport élaboré pour la DGS, intitulé Objectifs pour une politique nutritionnelle de santé publique pour la France, et constituent le socle du programme national nutrition-santé, orienté vers la population générale. L'objectif général de ce programme est l'amélioration de l'état nutritionnel, la réduction du risque de maladies chroniques, l'amélioration de l'état de santé et de la qualité de vie de la population générale.

Le fait d'atteindre ces objectifs ne constitue pas une fin en soi, mais le niveau de réduction des facteurs de risque retenus est considéré comme raisonnable et surtout atteignable dans une durée limitée, tout en permettant un impact réel significatif à plus long terme sur la morbidité et la mortalité.

Encadré

Objectifs nutritionnels prioritaires en termes de santé publique

Objectifs portant sur des modifications de la consommation alimentaire :

- augmentation de la consommation de fruits et légumes : réduction du nombre de petits consommateurs de fruits et légumes d'au moins $25 \%$; 
- augmentation de la consommation de calcium afin de réduire de $25 \%$ la population des sujets ayant des apports calciques en dessous des apports nutritionnels conseillés, associée à une réduction de $25 \%$ de la prévalence des déficiences en vitamine $D$;

- réduction de la moyenne des apports lipidiques totaux à moins de $35 \%$ des apports énergétiques journaliers, avec une réduction d'un quart de la consommation des acides gras saturés au niveau de la moyenne de la population (moins de $35 \%$ des apports totaux de graisses) ;

- augmentation de la consommation de glucides afin qu'ils contribuent à plus de $50 \%$ des apports énergétique journaliers, en favorisant la consommation des aliments sources d'amidon, en réduisant de $25 \%$ la consommation actuelle de sucres simples, et en augmentant de $50 \%$ la consommation de fibres ;

- réduction de la consommation d'alcool qui ne devrait pas dépasser $20 \mathrm{~g}$ d'alcool chez ceux qui consomment des boissons alcoolisées. Cet objectif vise la population générale et se situe dans le contexte nutritionnel (contribution excessive à l'apport énergétique) ; il n'est pas orienté sur la population des sujets présentant un problème d'alcoolisme chronique, redevable d'une prise en charge spécifique.

\section{Objectifs portant sur des modifications des marqueurs de l'état nutritionnel :}

- réduction de $5 \%$ de la cholestérolémie moyenne dans la population des adultes ;

- une réduction de $10 \mathrm{~mm}$ de $\mathrm{Hg}$ de la pression artérielle systolique chez les adultes ;

- une réduction de $20 \%$ de la prévalence du surpoids et de l'obésité (IMC $\left.>25 \mathrm{~kg} / \mathrm{m}^{2}\right)$ chez les adultes et une interruption de l'augmentation, particulièrement élevée au cours des dernières années, de la prévalence de l'obésité chez les enfants.

\section{Objectifs portant sur la modification de l'hygiène de vie en relation avec l'alimentation}

Augmentation de l'activité physique dans les activités de la vie quotidienne par une amélioration de $25 \%$ du pourcentage des sujets faisant, par jour, l'équivalent d'au moins $1 / 2 \mathrm{~h}$ de marche rapide (monter escalier à pied, faire les courses à pied). La sédentarité étant un facteur de risque de maladies chroniques doit être combattue chez l'enfant.

\section{Les objectifs nutritionnels spécifiques}

À côté de ces objectifs prioritaires qui concernent l'ensemble de la population, des objectifs spécifiques doivent être également retenus. Ils visent à résoudre certains problèmes nutritionnels observés chez différents groupes à risque (femmes enceintes et allaitantes, femmes en âge de procréer, enfants et adolescents, sujets âgés, situations de précarité, etc.) et portant spécifiquement sur certains nutriments (fer, folates, calcium, vitamine D) ou certains risques particuliers (carences spécifiques, malformations congénitales, allergies, etc.). La prise en compte de ces objectifs spécifiques peut associer diverses approches : recommandations nutritionnelles, utilisation de compléments ou de 
suppléments, enrichissement des aliments.

\section{Outils de santé publique nécessaires}

\section{Des apports nutritionnels conseillés régulièrement actualisés}

Le suivi permanent de la validité de ces références ainsi que la publication régulière d'une édition actualisée devront être mis en place.

\section{Un guide alimentaire national largement diffusé}

Ce document pratique et aisément compréhensible proposera un mode d'alimentation intégré, fondé sur les choix d'aliments et de comportements permettant à chaque individu de satisfaire les apports nutritionnels conseillés et, au niveau de la collectivité, d'atteindre les objectifs nutritionnels prioritaires de santé publique retenus. Sa diffusion doit s'inscrire dans une stratégie précise et définie à l'avance.

Des guides complémentaires pourront être élaborés pour les groupes concernés par les objectifs nutritionnels spécifiques (femmes enceintes, adolescents, sujets âgés, etc.).

\section{Mesures et actions de santé publique destinées à l'ensemble de la population}

Mesures politiques influençant les consommations alimentaires, la qualité nutritionnelle des aliments et l'activité physique

* Intégration de la dimension nutritionnelle dans toutes les mesures politiques touchant directement ou indirectement l'alimentation (politique agricole, sociale, réglementation, etc.).

* Développement de mesures législatives et politiques ayant un impact favorable sur les consommations et comportements alimentaires (lois, subventions et mesures fiscales incitatives).

* Adaptation de la réglementation dans le domaine nutritionnel aux objectifs de santé publique.

* Incitation et soutien aux initiatives des producteurs en matière de qualité nutritionnelle.

* Aménagements urbains favorisant l'activité physique dans la vie quotidienne.

* Mesures incitant les employeurs à favoriser l'activité physique des salariés ayant une activité sédentaire.

Programmes d'information et d'éducation autour des objectifs nutritionnels prioritaires et des objectifs spécifiques

Leur conception, leur mise en œuvre et leur évaluation nécessitent la mobilisation de tous les partenaires concernés : ministères et administrations (Santé, Agriculture, Économie et 
Finances, Éducation nationale, Recherche, Ville, Environnement, etc.), responsables et acteurs de santé publique régionaux, scientifiques et chercheurs impliqués dans la nutrition, associations de consommateurs, spécialistes en éducation pour la santé, sociologues, industriels, spécialistes en marketing, publicitaires, agences de communication, représentants des médias.

Les campagnes d'information " grand public " devront être développées sur une grande échelle : TV, radio, presse écrite nationale et régionale. Elles seront précédées de campagnes destinées aux professionnels de santé, afin qu'ils puissent être des relais efficaces de ces informations. Elles reposeront sur des messages simples, limités, clairs et spécifiques, déclinés en fonction des différents publics cibles. Elles devront viser à responsabiliser les consommateurs pour en faire des acteurs de leur santé. Elles porteront, à côté des objectifs à finalité alimentaire, sur l'activité physique dans la vie quotidienne (par exemple : campagnes de communication dans les transports urbains pour promouvoir l'intérêt de descendre à la station précédant celle initialement programmée; dans les établissements publics pour rappeler l'intérêt « santé » de monter à pied les escaliers, etc.).

Des centres-ressources régionaux, s'intégrant aux structures de santé publique existantes, devront être mis en place pour favoriser l'appropriation de ces programmes par les acteurs de terrain. Les associations de consommateurs devront également être associées à cette dynamique, tant au plan national qu'au niveau local.

Un soutien des médias est indispensable, notamment pour amplifier les campagnes d'information, mais aussi pour rétablir la vérité face à la diffusion d'informations induisant en erreur les consommateurs.

Des moyens doivent être mis en place pour garantir et crédibiliser la publicité des entreprises souhaitant communiquer de façon adéquate et soutenir les messages et produits alimentaires ayant une pertinence nutritionnelle (contribuant à atteindre les objectifs nutritionnels prioritaires). Parallèlement, il est indispensable de contrôler les allégations concernant certains produits présentant des pseudo-vertus nutritionnelles et de réprimer la publicité trompeuse.

\section{Actions en milieu scolaire et universitaire}

Différentes mesures ou actions doivent donc être associées dans ce secteur :

- intégration de la dimension alimentation/nutrition et notamment d'éléments du Guide alimentaire national (et d'un éventuel guide spécifique pour l'enfant), dans les programmes et manuels scolaires ;

- implication des services de promotion de la santé en faveur des élèves ;

- intégration des repas scolaires dans les processus d'éducation nutritionnelle et dans les moyens d'atteindre les objectifs nutritionnels prioritaires (adaptation des menus) ;

- mise en pratique des recommandations nutritionnelles officielles du GPEM-DA concernant 
les repas servis en milieu scolaire ;

- labellisation (en accord avec la charte mise au point par l'Institut français pour la nutrition) du matériel pédagogique destiné aux élèves produit par des firmes agro-alimentaires ;

- limitation des produits de grignotage dans les distributeurs automatiques situés dans ou à proximité des établissements scolaires, obligation d'intégrer des aliments ayant une valeur nutritionnelle adéquate (fruits, produits laitiers, produits céréaliers, etc.) ;

- obligation d'installer des fontaines d'eau fraîche dans les établissements scolaires et interdiction des distributeurs des boissons sucrées énergétiques (sodas) ;

- distribution gratuite de fruits (sous une forme séduisante pour les enfants) et de produits laitiers à la collation du matin ou au goûter de l'après-midi ;

- développement de l'éducation physique à l'école, dans l'optique de favoriser la gestion de la vie physique future.

\section{Actions au niveau de la restauration collective et des lieux de travail}

Les sociétés de restauration collective souhaitant s'associer à cette démarche s'engageront à proposer des menus labellisés " programme national nutrition-santé » et à participer aux campagnes d'information répondant à un cahier des charges préalablement défini.

\section{Implication forte des industriels de l'agro-alimentaire}

Les objectifs nutritionnels de santé publique ne peuvent être atteints sans une collaboration étroite avec les industriels de l'agro-alimentaire, qui doit être envisagée à différents niveaux dans le cadre de :

- l'amélioration des produits alimentaires mis à la disposition des consommateurs, notamment des aliments dont la consommation contribue à atteindre les objectifs de santé publique ;

- la communication ou la promotion autour de ces aliments ;

- l'information du public sur les objectifs nutritionnels prioritaires.

Deux mesures spécifiques doivent permettre de favoriser l'adhésion des industriels.

* La création d'un label officiel " programme nutrition-santé »

Ce label sera matérialisé sous forme d'un logo, qui devra être largement médiatisé pour être connu et reconnu par la population générale. Il sera délivré sur demande pour tout produit alimentaire répondant à un cahier des charges strict et prédéfini, et considéré comme contribuant aux objectifs nutritionnels prioritaires.

Ce logo permettra également de labelliser la communication (campagnes publicitaires) des produits alimentaires faisant allusion directement ou indirectement à une valeur santé 
intrinsèque ou ajoutée, à condition que cette communication présente une réelle pertinence nutritionnelle.

* La possibilité d'utilisation d'allégations " nutritionnelles " issues du " programme nutrition-santé " sur les produits alimentaires et dans la communication relative à ces produits. Les allégations autorisées porteront sur les données génériques reprenant les objectifs nutritionnels prioritaires (et non directement sur les produits eux-mêmes).

Une charte de bonne pratique mise à la disposition des industriels de l'agro-alimentaire devrait faciliter leurs demandes de labellisation et/ou d'allégations. Une réflexion développée avec les industriels de l'agro-alimentaire et les consommateurs devrait permettre de déboucher sur un principe d'étiquetage informatif.

\section{Actions au niveau du système de soins}

Les acteurs du système de soins occupent une place privilégiée auprès des consommateurs et leur soutien est essentiel, à la fois pour relayer auprès de la population les campagnes d'information nutritionnelle, conforter les recommandations et assurer la prise en charge précoce des problèmes nutritionnels.

Le programme national nutrition-santé doit aussi être l'occasion d'améliorer la détection et la prise en charge des maladies nutritionnelles par le développement :

- d'un outil d'évaluation clinique de l'état nutritionnel ;

- de recommandations pour la pratique clinique concernant la prévention ;

- de démarches d'éducation du patient ;

- par le diagnostic et le traitement des principales maladies liées à la nutrition ;

- par la mise en place de structures d'évaluation de l'alimentation hospitalière.

\section{Actions au niveau des supports artistiques, culturels, sportifs et des personnalités politiques}

Le soutien du programme national nutrition-santé par les différents supports artistiques, culturels et sportifs, et des personnalités politiques (maires, conseillers généraux et régionaux, députés, sénateurs, ministres) pouvant favoriser la promotion des objectifs nutritionnels prioritaires devra être activement recherché.

Mise en place à la DGS d'un comité d'appui pour la mise en place et le suivi de la politique nutritionnelle

\section{Mesures et actions de santé publique spécifiques}

Ces mesures porteront sur :

- la lutte contre la carence en fer pendant la grossesse ; 
- I'amélioration du statut en folates des femmes en âge de procréer, notamment en cas de désir de grossesse ;

- la promotion de l'allaitement maternel ;

- l'amélioration du statut en fer, en calcium et en vitamine D des enfants et des adolescents ;

- l'amélioration du statut en calcium et en vitamine D des personnes âgées ;

- la lutte contre la dénutrition des personnes âgées ;

- la lutte contre les déficiences vitaminiques et minérales et les dénutritions chez les sujets en grande situation de précarité ;

- la lutte contre les déficiences vitaminiques et minérales chez les sujets suivant des régimes restrictifs ;

- des mesures visant la prise en compte des problèmes d'allergies alimentaires.

\section{Actions de formation}

Les formations médicales et paramédicales. La formation initiale et continue des médecins doit intégrer l'alimentation de l'homme " sain ", la prévention nutritionnelle des grandes maladies chroniques, la nutrition thérapeutique. Ces objectifs concernent également les infirmiers et les kinésithérapeutes.

La formation des enseignants, des formateurs et des professions relais. Le corps enseignant doit pouvoir non seulement assurer les enseignements prévus dans le cadre des programmes, mais aussi relayer de façon efficace, rigoureuse et complémentaire les messages préventifs en matière de nutrition et d'alimentation. II est donc indispensable d'intégrer la dimension alimentation/nutrition dans les programmes de formation initiale et continue de ces professionnels. Une telle formation est également indispensable pour les gestionnaires et le personnel d'encadrement de restauration scolaire et plus généralement, de restauration collective.

La formation des " diététiciens " et des professionnels de l'industrie agro-alimentaire. II semble nécessaire de mettre en place une formation correspondant à un nouveau métier de diététicien nutritionniste. II faut également former à la nutrition humaine les professionnels destinés à travailler dans l'industrie agro-alimentaire.

\section{Promotion de la recherche en nutrition humaine}

La recherche en nutrition humaine souffre en France de sa dispersion et de l'absence d'une politique globale définissant ses objectifs et cernant les limites de son domaine d'action. II est indispensable de promouvoir des recherches au sein des organismes de recherche (Inserm, Inra, CNRS, etc.) et des universités dans le domaine des déterminants et de l'évaluation de l'état nutritionnel, de la compréhension des relations entre alimentation et 
santé, de l'évaluation des actions de prévention nutritionnelle. Il est indispensable de développer des moyens visant à créer, soutenir et mieux financer les unités, centres et équipes de recherche ou universitaires dédiées à la recherche en nutrition et notamment en nutrition humaine et dans ses applications en santé publique.

Des programmes de recherche incitatifs dans les domaines de l'étude des relations entre l'alimentation et la santé devraient permettre de stimuler et soutenir financièrement les travaux dans ces domaines. La création d'un réseau technologique de nutrition, sous l'égide du ministère de la Recherche, associant les pouvoirs publics, les chercheurs, les industriels de l'agro-alimentaires et les consommateurs devrait permettre de définir un plan national et de hiérarchiser les priorités de recherche. Par cohérence aux objectifs de santé publique, une priorité devra être donnée aux recherches dans les domaines des relations alimentation-cancer, alimentation-maladies cardiovasculaires, alimentation-obésité et alimentation-ostéoporose.

\section{Moyens de surveillance de l'état nutritionnel de la population}

L'ajustement du programme national nutrition-santé à l'évolution de la situation nutritionnelle de la population ainsi que son évaluation nécessitent la mise en place d'un dispositif permettant de centraliser les données existantes et de collecter régulièrement de façon transversale des données sur un échantillon représentatif de la population, de disposer des moyens d'évaluation de l'état nutritionnel de la population et des groupes de population à risque de problèmes nutritionnels.

Le nombre de produits alimentaires actuellement commercialisés est tout à fait considérable, et l'innovation dans ce domaine est très rapide. Il est donc essentiel de mettre en œuvre les moyens nécessaires pour que les tables de composition des aliments soient actualisées et enrichies en permanence.

\section{CONCLUSION}

La mise en place d'une politique nutritionnelle apparaît comme une grande priorité de santé publique en France, comme dans l'ensemble de l'Europe. Outre les immenses enjeux humains, sociaux et économiques, les relations entre l'alimentation et la santé ont plusieurs particularités.

* Elles concernent l'ensemble de la population. Si certains groupes de populations connaissent des risques spécifiques, tous les individus et tout au long de leur vie sont directement confrontés, au travers des aliments qu'ils consomment quotidiennement et/ou de leurs habitudes alimentaires, à un déterminant majeur de l'état de santé. Par ses choix alimentaires et son mode de vie, chaque consommateur s'expose, souvent sans en avoir conscience, à d'éventuels facteurs de risque ou de protection vis-à-vis de certaines maladies chroniques. II est donc un acteur essentiel pouvant intervenir directement sur un déterminant majeur de sa santé.

* Elles reposent sur des connaissances scientifiques bien documentées. Les travaux épidémiologiques, cliniques et expérimentaux fournissent des informations scientifiques suffisamment fiables pour permettre d'identifier, de façon consensuelle, des facteurs 
nutritionnels impliqués dans le risque ou la protection vis-à-vis de certains grands problèmes de santé publique.

* Elles concernent des facteurs de risque (ou de protection) bien identifiés sur lesquels il est possible d'intervenir. S'il est difficile d'agir sur la composante génétique impliquée dans le déterminisme des grandes maladies chroniques (cancers, maladies cardiovasculaires, obésité, ostéoporose, etc.), les facteurs alimentaires et nutritionnels sont particulièrement accessibles. Par ailleurs, leur prise en compte peut se faire non pas sur le mode de l'interdit, mais sur celui de la promotion de comportements favorables à la santé.

* Elles ont un impact important sur l'incidence des grandes maladies chroniques. En agissant sur l'alimentation (et l'activité physique), il est possible de réduire, à court terme, l'exposition à certains facteurs de risque et de promouvoir certains facteurs de protection, laissant augurer une réduction de la morbidité et à plus long terme de la mortalité.

* Elles impliquent, autour du consommateur, de multiples acteurs du champ de la nutrition (professionnels de santé, acteurs de santé publique, secteur agro-alimentaire, restauration collective, milieux scolaires et universitaires, formateurs, enseignants, chercheurs, communicants, législateurs, monde associatif, médias, etc.), de multiples structures politiques (Santé, Agriculture, Éducation nationale, Recherche, Économie et Finances, Jeunesse et Sports, Ville, Transports, Culture, etc.) et institutions (InVS, Afssa, DGS, DGAL, DGCCRF, Inra, Inserm, CFES, etc.). Au cœur des actions développées avec l'ensemble des acteurs concernés, une réelle implication des industriels de l'agro-alimentaire doit permettre d'avoir un impact majeur sur les aliments eux-mêmes et sur les choix des aliments par les consommateurs.

* Elles ont des références en termes de faisabilité. L'efficacité de la lutte contre les facteurs de risque cardiovasculaire mise en place en Finlande au cours des vingt dernières années illustre la faisabilité de projets nationaux ambitieux et leur efficacité à réduire la morbidité et la mortalité.

À la suite de ce rapport, un " programme national nutrition-santé » assurant la promotion de la nutrition et de l'activité physique a été annoncé le 13 décembre 2000 par M. Lionel Jospin, Premier ministre, et lancé le 24 janvier 2001 par Mme Dominique Gillot, secrétaire d'État à la Santé. Ce Programme bâti sur cinq ans reprend un nombre important de recommandations du rapport du HCSP.

\section{REFERENCES}

1. Rapport du HCSP. Pour une politique nutritionnelle de santé publique : enjeux et propositions. Editions ENSP, septembre $2000 ; 250$ p. 